\title{
A note on geometric 3-hypergraphs
}

\author{
Andrew Suk*
}

November 22, 2018

\begin{abstract}
In this note, we prove several Turán-type results on geometric hypergraphs. The two main theorems are 1) Every $n$-vertex geometric 3-hypergraph in the plane with no three strongly crossing edges has at most $O\left(n^{2}\right)$ edges, 2) Every $n$-vertex geometric 3-hypergraph in 3-space with no two disjoint edges has at most $O\left(n^{2}\right)$ edges. These results support two conjectures that were raised by Dey and Pach, and by Akiyama and Alon.
\end{abstract}

\section{Introduction}

A geometric r-hypergraph $H$ in d-space is a pair $(V, E)$, where $V$ is a set of points in general position in Euclidean $d$-space, and $E$ is a set of closed $(r-1)$-dimensional simplices (edges) induced by some $r$-tuple of $V$. The sets $V$ and $E$ are called the vertex set and edge set of $H$, respectively. Two edges in $H$ are crossing if they are vertex disjoint and have a point in common. Notice that if $k$ edges are pairwise crossing, it does not imply that they all have a point in common. Hence we say that $H$ contains $k$ strongly crossing edges if $H$ contains $k$ vertex disjoint edges that all share a point in common. See Figure 1.

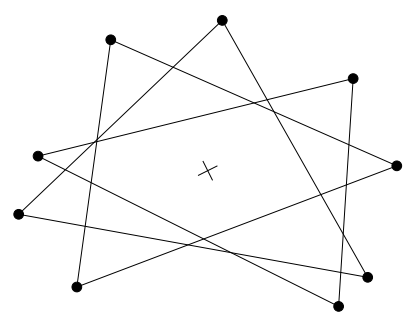

(a) Three strongly crossing edges.

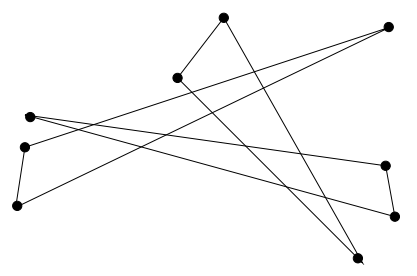

(b) Three pairwise crossing edges with an empty intersection.

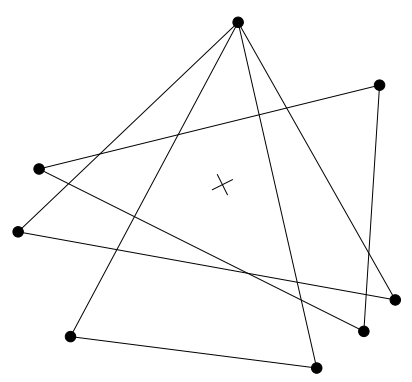

(c) Three edges not strongly crossing since two share a vertex.

Figure 1: Three edges of a geometric 3-hypergraph in the plane.

A direct application of the colored Tverberg theorem (see [3, 20]) gives

${ }^{*}$ Courant Institute, New York and EPFL, Lausanne. Email: suk@cims.nyu.edu. The author gratefully acknowledges the support from the Swiss National Science Foundation, Grant No. 200021-125287/1. 
Theorem 1.1. Let $e x_{d}\left(S C_{k}^{d+1}, n\right)$ denote the maximum number of edges an n-vertex geometric $(d+1)$-hypergraph in $d$-space has with no $k$ strongly crossing edges. Then

$$
e x_{d}\left(S C_{k}^{d+1}, n\right)=O\left(n^{d+1-\frac{1}{(2 k-1)^{d}}}\right) .
$$

Dey and Pach [5] showed that $e x_{d}\left(S C_{2}^{d+1}, n\right)=\Theta\left(n^{d}\right)$, and conjectured $e x_{d}\left(S C_{k}^{d+1}, n\right)=\Theta\left(n^{d}\right)$ for every fixed $d$ and $k$. The lower bound can easily be seen by taking all edges with a vertex in common. The main motivation for their conjecture is for deriving upper bounds on the maximum number of $k$-sets of an $n$-point set in $\mathbb{R}^{d}$. See $[12$ for more details. In this note, we settle the DeyPach conjecture for geometric 3-hypergraphs in the plane with no three strongly crossing edges, and improve the upper bound of $e x_{2}\left(S C_{k}^{3}, n\right)$.

Theorem 1.2. $\mathrm{ex}_{2}\left(S C_{3}^{3}, n\right)=\Theta\left(n^{2}\right)$.

Theorem 1.3. For fixed $k \geq 4$, ex $x_{2}\left(S C_{k}^{3}, n\right) \leq O\left(n^{3-\frac{1}{k}}\right)$.

As a related result, Akiyama and Alon [2] used the Borsuk-Ullam Theorem [4] to show the following.

Theorem 1.4. Let $\operatorname{ex}_{d}\left(D_{k}^{d}, n\right)$ denote the maximum edges that an n-vertex geometric d-hypergraph in $d$-space has with no $k$ pairwise disjoint edges. Then

$$
e x_{d}\left(D_{k}^{d}, n\right) \leq n^{d-(1 / k)^{d-1}}
$$

They conjecture that for every fixed $d$ and $k, e x_{d}\left(D_{k}^{d}, n\right)=\Theta\left(n^{d-1}\right)$. Again the lower bound can easily be seen by taking all edges with a vertex in common. Pach and Töröcsik [15] showed that $\operatorname{ex}_{2}\left(D_{k}^{2}, n\right)=O\left(k^{4} n\right)$, which was later improved to $O\left(k^{2} n\right)$ by Tóth [17]. Here we settle the Akiyama-Alon conjecture for geometric 3-hypergraphs in 3-space with no two disjoint edges.

Theorem 1.5. $e x_{3}\left(D_{2}^{3}, n\right)=\Theta\left(n^{2}\right)$.

For clarity of the proofs, we do not make any attempts to optimize the constants.

\section{Strongly crossing edges in the plane}

In this section we will prove Theorems 1.2 and 1.3. Recall that a geometric graph is a graph drawn in the plane with vertices represented by points and edges by straight line segments connecting the corresponding pairs. Recently Ackerman [1] showed the following.

Lemma 2.1. Let $G=(V, E)$ be an n-vertex geometric graph in the plane with no four pairwise crossing edges. Then $|E(G)| \leq O(n)$.

We note that Lemma 2.1 holds for topological graphs. Before we give the proofs, we will introduce some terminology. Consider a family $\mathcal{S}=\left\{s_{1}, \ldots, s_{k}\right\}$ of pairwise crossing segments in the plane, and let $\mathcal{L}=\left\{l_{1}, \ldots, l_{k}\right\}$ be a family of lines such that $l_{i}$ is the line supported by segment $s_{i}$. Recall that the level of a point $x \in \cup \mathcal{L}$ is defined as the number of lines of $\mathcal{L}$ lying strictly below $x$. We 

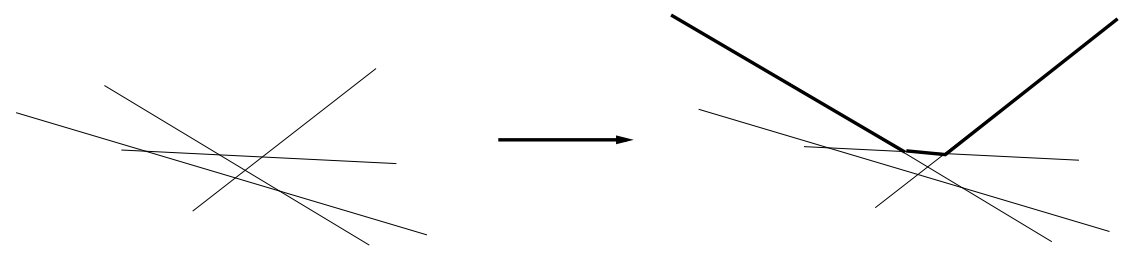

Figure 2: The top level of four pairwise crossing segments is drawn thick.

define the top level of $\mathcal{L}$ as the closure of the set of points in $\cup \mathcal{L}$ with level $k-1$. We define the top level of $\mathcal{S}$ to be the top level of $\mathcal{L}$. See Figure 2, Notice that $L$ is a (not strictly) convex function.

For each edge $t$ in a geometric 3-hypergraph in the plane, we define its base as the side with the longest $x$-projection. We define the other two sides of $t$ as its left and right side. See Figure 2. Notice that every edge in a geometric 3-hypergraph is incident to a vertex that lies strictly above or below its base. We are now ready to prove Theorem 1.2 .

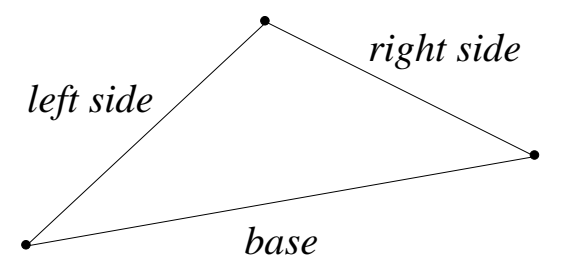

Figure 3: The base, left side, and right side.

Proof of Theorem 1.2. Let $H=(V, E)$ be an $n$-vertex geometric 3-hypergraph in the plane with no three strongly crossing edges. We can assume that $|E(H)| \geq 20 n^{2}$ (since otherwise we would be done) and at most $|E(H)| / 2$ edges in $H$ are incident to a vertex that lies strictly below its base. We will discard all such edges, leaving us with at least $|E(H)| / 2$ edges left. Let $E_{u v}$ be the set of edges in $H$ with base $u v$. We discard all sets $E_{u v}$ for which $\left|E_{u v}\right| \leq|E(H)| /\left(2 n^{2}\right)$. Since we have thrown away at most $|E(H)| / 4$ edges in this process, we have at least $|E(H)| / 4$ edges left. Therefore $\left|E_{u v}\right|=0$ or $\left|E_{u v}\right| \geq|E(H)| /\left(2 n^{2}\right) \geq 10$.

Now let $G_{v}=(V, E)$ denote the geometric graph with $V\left(G_{v}\right)=V(H)$ and $x y \in E\left(G_{v}\right)$ if $\operatorname{conv}(x \cup y \cup v) \in E(H)$ with base $x y$.

Observation 2.2. $G_{v}$ does not contain four pairwise crossing edges (bases).

Proof. For sake of contradiction, suppose $G_{v}$ contains four pairwise crossing edges $b_{1}, b_{2}, b_{3}, b_{4} \in$ $E\left(G_{v}\right)$. Then $v$ lies above $b_{i}$ for all $i$. Let $L$ denote the top level of the arrangement $\mathcal{S}=$ $\left\{b_{1}, b_{2}, b_{3}, b_{4}\right\}$. Now the proof falls into three cases.

Case 1. Suppose $L$ intersects exactly two members of $\mathcal{S}$, say bases $b_{1}$ and $b_{2}$ (in order from left to right along $L$ ). Let $p$ be the intersection point of $b_{1}$ and $b_{2}$. Then the vertical line through $p$ must intersect $b_{3}$ below $p$. Moreover, since segments $b_{1}$ and $b_{3}$ cross, $v$ and the right-endpoint of $b_{3}$ must lie on the same half-plane generated by the line supported by $b_{1}$. Likewise, $v$ and the left-endpoint of $b_{3}$ must lie on the same half-plane generated by the line supported by $b_{2}$. Therefore 
$p \in \operatorname{conv}\left(v \cup b_{3}\right)$. See Figure $4(\mathrm{a})$. Since $\left|E_{b_{1}}\right|,\left|E_{b_{2}}\right| \geq 10$, there exists vertices $x, y \in V(H)$ such that $\operatorname{conv}\left(v \cup b_{3}\right), \operatorname{conv}\left(x \cup b_{1}\right), \operatorname{con} v\left(y \cup b_{2}\right)$ are three (vertex disjoint) strongly crossing edges in $H$ and we have a contradiction.

Case 2. Suppose $L$ intersects exactly three members of $\mathcal{S}$, say bases $b_{1}, b_{2}, b_{3}$ (in order from left to right along $L$ ). Now $b_{4}$ must intersect $b_{2}$ either to the left or right of $b_{2} \cap L$. Without loss of generality, we can assume that $b_{4}$ intersects $b_{2}$ to the right of $b_{2} \cap L$. Let $p$ be the intersection point of segments $b_{2}$ and $b_{3}$. By the same argument as above, $p \in \operatorname{conv}\left(v \cup b_{4}\right)$. See Figure 4(b). Since $\left|E_{b_{1}}\right|,\left|E_{b_{2}}\right| \geq 10$, there exists vertices $x, y \in V(H)$ such that conv $\left(v \cup b_{4}\right), \operatorname{conv}\left(x \cup b_{1}\right), \operatorname{conv}\left(y \cup b_{2}\right)$ are three strongly crossing edges in $H$ and we have a contradiction.

Case 3. Suppose $L$ intersects $b_{1}, b_{2}, b_{3}, b_{4}$ in order from left to right along $L$. Let $p$ be the intersection point of segments $b_{2}$ and $b_{3}$, and let $l$ be the vertical line through $v$. Since the right endpoint of $b_{4}$ lies to the right of $l$, and the left endpoint of $b_{1}$ lies to the left of $l$, we have $p \in \operatorname{conv}\left(v \cup b_{1}\right) \cup \operatorname{conv}\left(v \cup b_{4}\right)$. Therefore, either conv $\left(v \cup b_{1}\right)$ or $\operatorname{con} v\left(v \cup b_{4}\right)$ (say conv $\left.\left(v \cup b_{1}\right)\right)$ contains $p$. See Figure 4(c). Since $\left|E_{b_{2}}\right|,\left|E_{b_{3}}\right| \geq 10$, there exists vertices $x, y \in V(H)$ such that conv $\left(v \cup b_{1}\right)$, conv $\left(x \cup b_{2}\right), \operatorname{conv}\left(y \cup b_{3}\right)$ are three strongly crossing edges in $H$ and we have a contradiction.

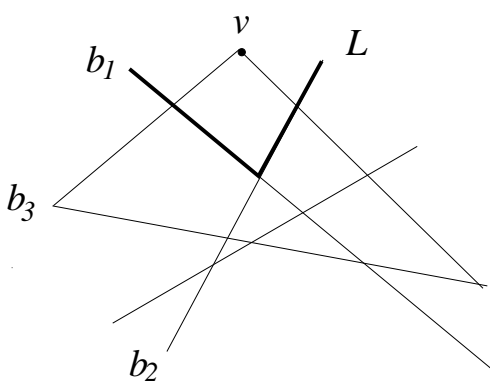

(a) Case 1.

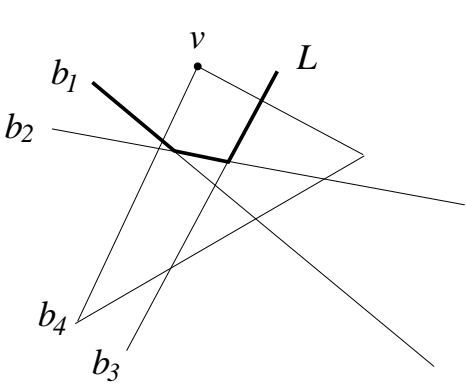

(b) Case 2 .

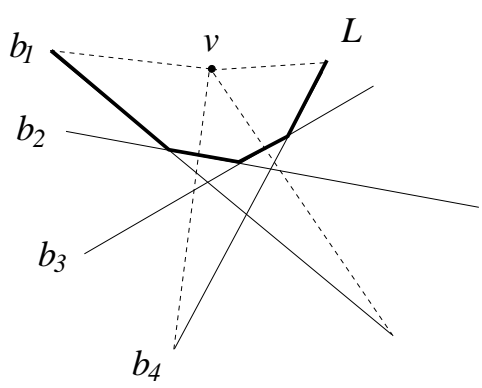

(c) Case 3 .

Figure 4: Three cases.

Therefore by Lemma 2.1, $\left|E\left(G_{v}\right)\right| \leq O(n)$ for every vertex $v \in V(H)$. Hence

$$
\frac{|E(H)|}{4} \leq \sum_{v \in V(H)}\left|E\left(G_{v}\right)\right|=O\left(n^{2}\right),
$$

which implies $|E(H)|=O\left(n^{2}\right)$.

Before we prove Theorem [1.3, we will need the following lemma due to Valtr [18].

Lemma 2.3. Let $G=(V, E)$ be an n-vertex geometric graph in the plane such that all of the edges in $G$ intersect the $y$-axis. If $G$ does not contain $k$ pairwise crossing edges, then $|E(G)| \leq c_{k} n$ where $c_{k}$ depends only on $k$.

Proof of Theorem 1.3. Let $H$ be an $n$-vertex geometric 3-hypergraph in the plane with no $k$ strongly crossing edges for $k \geq 4$. Just as before, we can assume at most $|E(H)| / 2$ of the edges in 
$H$ are incident to a vertex that lies strictly below its base. We discard all such edges, leaving us with at least $|E(H)| / 2$ edges left in $H$. Now we make the following observation.

Observation 2.4. Suppose $b_{1}, \ldots, b_{k}$ are $k$ pairwise crossing bases and $v_{1}, \ldots, v_{k} \in V(H)$ such that $\operatorname{conv}\left(v_{i} \cup b_{j}\right) \in E(H)$ with base $b_{j}$ for all $i, j$. Then $H$ contains $k$ strongly crossing edges.

Proof. Let $L$ denote the top level of the segment arrangement $\mathcal{S}=\left\{b_{1}, \ldots, b_{k}\right\}$ and assume that $b_{1}, \ldots, b_{k}$ are ordered by increasing slopes. See Figure 5 .

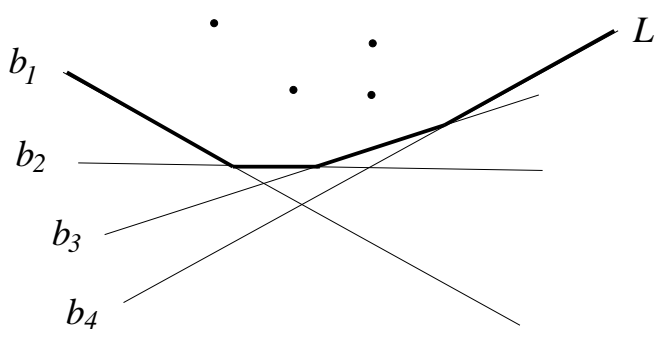

Figure 5: Arrangement of $b_{1}, b_{2}, b_{3}, b_{4}$.

Now we define edges $t_{1}, t_{2}, \ldots, t_{k} \in E(H)$ as follows. Among the $k$ edges conv $\left(b_{1} \cup v_{1}\right), \operatorname{conv}\left(b_{1} \cup\right.$ $\left.v_{2}\right), \ldots, \operatorname{conv}\left(b_{1} \cup v_{k}\right) \in E(H)$, (with slight abuse of notation) let $t_{1}=\operatorname{conv}\left(b_{1} \cup v_{1}\right)$ be the edge whose right side has the rightmost intersection with $L$. Then among the $k-1$ edges $\operatorname{conv}\left(b_{2} \cup\right.$ $\left.v_{2}\right)$, conv $\left(b_{2} \cup v_{3}\right), \ldots, \operatorname{conv}\left(b_{2} \cup v_{k}\right)$, (again with slight abuse of notation) let $t_{2}=\operatorname{conv}\left(b_{2} \cup v_{2}\right)$ be the edge whose right side has the rightmost intersection with $L$. We continue this procedure until we have $k$ edges $t_{1}, t_{2}, \ldots, t_{k}$. Clearly these $k$ edges are vertex disjoint.

Now notice that $\left(t_{i} \cap L\right) \cap\left(t_{j} \cap L\right) \neq \emptyset$ for all pairs $i, j$. Indeed for sake of contradiction, suppose there exists two edges $t_{i}$ and $t_{j}$ for $i<j$ such that either $t_{i} \cap L$ lies completely to the left of $t_{j} \cap L$ or vice versa. See Figure 6 .
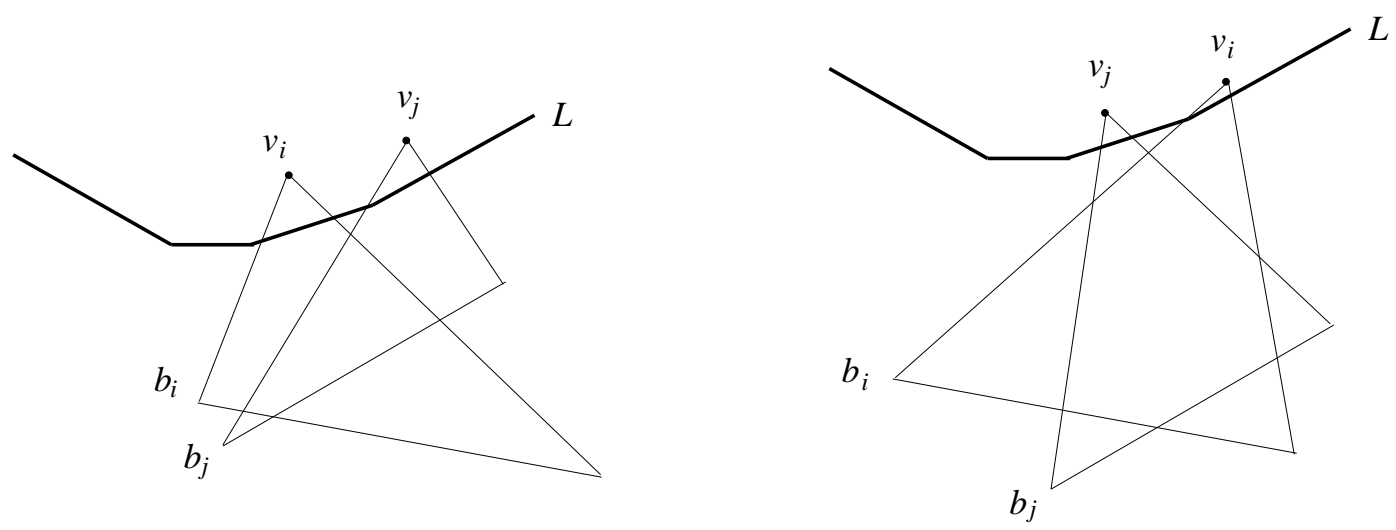

Figure 6: Assume $\left(t_{i} \cap L\right) \cap\left(t_{j} \cap L\right)=\emptyset$.

Case 1. Suppose $t_{i} \cap L$ lies completely to the left of $t_{j} \cap L$. Then the vertical line through $v_{j}$ intersects the right side of $t_{i}$ below $v_{j}$. Therefore the right side of $\operatorname{conv}\left(b_{i} \cup v_{j}\right)$ intersects $L$ more to the right than the right side of $t_{i}=\operatorname{conv}\left(b_{i} \cup v_{i}\right)$ does. This contradicts the definition of $t_{i}$ and $t_{j}$. 
Case 2. Suppose $t_{i} \cap L$ lies completely to the right of $t_{j} \cap L$. Then there exists a base $b_{s}$ that has a point $p$ on $L$ between $t_{i} \cap L$ and $t_{j} \cap L$. Base $b_{s}$ must

1. lie below $v_{i}$ and $v_{j}$,

2. cross $b_{i}$ and $b_{j}$, and

3. contain point $p$.

However this impossible by the following argument. Let $l$ be the vertical line through $p$. Clearly $l$ intersects $b_{i}$ and $b_{j}$. Since $b_{s}$ lies below $v_{i}$ and $v_{j}, b_{s}$ must intersect $b_{j}$ to the left of $l$, and intersect $b_{i}$ to the right of $l$. Since $b_{s}$ intersects $b_{j}$ to the left of $l$, the slope of $b_{s}$ must be greater than the slope of $b_{j}$. However since the slope of $b_{i}$ is less than the slope of $b_{j}$, this implies that $b_{s}$ cannot intersect $b_{i}$ to the right of $l$. Hence we have a contradiction.

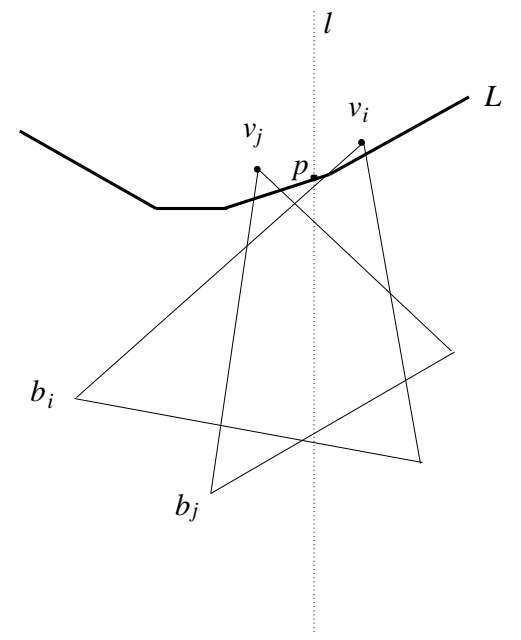

Figure 7: Case 2.

Since $\left(t_{i} \cap L\right) \cap\left(t_{j} \cap L\right) \neq \emptyset$ for every $i, j \in\{1,2, . ., k\}$, by Helly's Theorem [6] $t_{1}, \ldots, t_{k}$ has a nonempty intersection on $L$.

Notice that no $k$ points in $V(H)$ have $c_{k} n$ bases in common. Indeed, otherwise the vertical line through any of these $k$ points would intersect all $c_{k} n$ bases, and by Lemma 2.3 there would be $k$ pairwise crossing bases. By Observation 2.4, we would have $k$ strongly crossing edges.

Now let $G=(A \cup B, E)$ be a bipartite graph where $A=V(H)$ and $B=V^{2}(H)$, such that $(v, x y) \in E(G)$ if $\operatorname{conv}(x \cup y \cup v) \in E(H)$ with base $x y$. Since $G$ does not contain $K_{k, c_{k} n}$ as a subgraph, we can use the following well known result of Kövári, Sós, Turán [10].

Theorem 2.5. If $G=(A \cup B, E)$ is a bipartite graph with $|A|=n$ and $|B|=m$ containing no subgraph $K_{r, s}$ with the $r$ vertices in $A$ and the $s$ vertices in $B$, then

$$
|E(G)| \leq(s-1)^{1 / r} n m^{1-1 / r}+(r-1) m .
$$


By plugging in the values $m=n^{2}, r=k, s=c_{k} n$ into Theorem 2.5, we obtain

$$
\frac{|E(H)|}{2} \leq|E(G)| \leq O\left(n^{3-\frac{1}{k}}\right) .
$$

Hence

$$
|E(H)| \leq O\left(n^{3-\frac{1}{k}}\right)
$$

\subsection{Convex geometric 3-hypergraphs}

In the case when the vertices are in convex position in the plane, extremal problems on geometric 3-hypergraphs become easier due to the linear ordering of its vertices. The proof of Observation 2.4 can be copied almost verbatim to conclude the following.

Observation 2.6. Let $H=(V, E)$ be a geometric 3-hypergraph in the plane with vertices in convex position. Suppose $H$ contains $k$ edges of the form $t_{i}=\operatorname{conv}\left(x_{i} \cup y_{i} \cup z_{i}\right)$, such that the vertices $\left(x_{1}, \ldots, x_{k}, y_{1}, \ldots, y_{k}, z_{1}, \ldots, z_{k}\right)$ appear in clockwise order along the boundary of their convex hull. Then $t_{1}, \ldots, t_{k}$ are $k$ strongly crossing edges.

Marcus and Klazar [9] extended the Marcus-Tardos theorem [13] by showing that the number of 1-entries in a $r$-dimensional $(0,1)$-matrix with side length $n$ which avoids an $r$-dimensional permutation matrix is $O\left(n^{r-1}\right)$. As pointed out by Marcus and Klazar, it is not difficult to modify their proof to obtain an $O\left(n^{r-1}\right)$ bound on the number of edges in an ordered $n$-vertex $r$-uniform hypergraph that does not contain a fixed ordered matching. Hence by Observation 2.6, we can conclude the following.

Theorem 2.7. Let $H=(V, E)$ be a geometric 3-hypergraph in the plane with vertices in convex position. If $H$ does not contain $k$ strongly crossing edges, then $|E(H)| \leq c_{k} n^{2}$ where $c_{k}$ is a constant that depends only on $k$.

\section{Disjoint edges in 3-space}

In this section, we will prove Theorem 1.5. Recall that two edges in a geometric graph are parallel if they are the opposite edges of a convex quadrilateral. Katchalski and Last [7] and Pinchasi [16] showed that all $n$-vertex geometric graphs with more than $2 n-2$ edges contain two parallel edges. By following Pinchasi's argument almost verbatim, one can prove the following.

Lemma 3.1. Let $G$ be a graph drawn on the unit sphere $S$ with vertices represented as points such that no three lie on a great circle, and edges uv $\in E(G)$ are drawn as arcs along the great circle containing points $u$ and $v$ of length less than $\pi$ (the shorter arc). We say that edges $e_{1}, e_{2} \in E(G)$ are avoiding if the great circle supported by $e_{1}$ is disjoint to $e_{2}$, and the great circle supported by $e_{2}$ is disjoint from $e_{1}$. If $|E(G)|>2 n-2$, then $G$ contains two avoiding edges. 
Proof of Theorem 1.5. Let $H=(V, E)$ be an $n$-vertex geometric 3-hypergraph in 3-space with no two disjoint edges. Fix a pair of vertices $u, v \in V(H)$, and just consider the edges $E_{u v}=\{t \in$ $E(H): u, v$ are vertices of $t\}$. We color $t \in E_{u v}$ red if all of the members of $E_{u v}$ lie in one of the closed half-spaces generated by the plane supported by $t$. Notice that there are at most two red edges in $E_{u v}$. Repeat this procedure for each pair of vertices, which will leave us with at most $n^{2}$ red edges in the end. Color the remaining edges blue, and let $d_{b}(v)$ denote the number of blue edges incident to $v$. Then we have

$$
\sum_{v \in V(H)} d_{b}(v) \geq 3 E(H)-3 n^{2}
$$

Therefore, there exists a vertex $v$ incident to at least $\left(3|E(H)|-3 n^{2}\right) / n$ blue edges. Now consider a small 2-dimensional sphere $S^{2}$ centered at $v$. Then the intersection of $S^{2}$ and the blue edges incident to $v$ forms a graph $G$ with at most $n$ vertices and at least $\left(3 E(H)-3 n^{2}\right) / n$ edges.

If $\left(3|E(H)|-3 n^{2}\right) / n>2 n-2$, then by Lemma 3.1 we know that $G$ contains two avoiding edges $x y$ and $w z$. Let $h$ be the plane supported by the blue edge $\operatorname{conv}(w \cup z \cup v) \in E(H)$. Then the blue edge $\operatorname{conv}(x \cup y \cup v)$ must lie in one of the closed half-spaces generated by the plane $h$. Since $\operatorname{conv}(w \cup z \cup v)$ is blue, there must be a red edge $\operatorname{conv}(w \cup z \cup p)$ such that $h$ separates it from $\operatorname{conv}(x \cup y \cup v)$. Hence $\operatorname{conv}(x \cup y \cup v)$ and $\operatorname{conv}(w \cup z \cup p)$ are disjoint and we have a contradiction. See Figure 8. Therefore $\left(3|E(H)|-3 n^{2}\right) / n \leq 2 n-2$, which implies $|E(H)| \leq O\left(n^{2}\right)$.

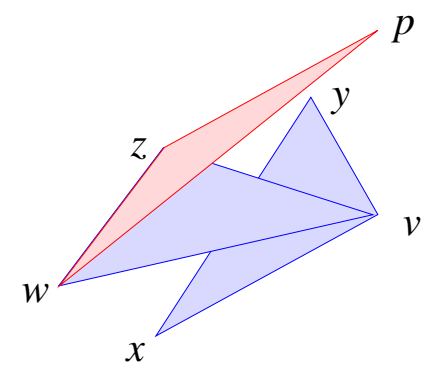

Figure 8: Disjoint edges conv $(w \cup z \cup p)$ and $\operatorname{conv}(x \cup y \cup v)$.

\section{Remarks}

By applying the Abstract Crossing Lemma (see [19]) to Theorem [1.2, every $n$-vertex geometric 3-hypergraph $H$ in the plane has either $O\left(n^{2}\right)$ edges or $\Omega\left(|E(H)|^{7} / n^{12}\right)$ triples that have a point in common. In the latter case, by the fractional Helly theorem [8] this implies one can always find a point inside at least $\Omega\left(|E(H)|^{5} / n^{12}\right)$ edges of $H$. However, this is not as strong as the

$$
\Omega\left(\frac{|E(H)|^{3}}{n^{6} \log ^{2} n}\right)
$$

bound obtained by Nivasch and Sharir [14]. 


\section{References}

[1] Ackerman, E.: On the maximum number of edges in topological graphs with no four pairwise crossing edges. In Proceedings of the Twenty-Second Annual Symposium on Computational Geometry (Sedona, Arizona, USA, June 05 - 07, 2006). SCG '06. ACM, New York, NY, 259-263.

[2] Akiyama, J. and Alon, N.: Disjoint simplices and geometric hypergraphs. In Proceedings of the Third international Conference on Combinatorial Mathematics (New York City, New York, United States). G. S. Bloom, R. L. Graham, and J. Malkevitch, Eds. New York Academy of Sciences, New York, NY, 1-3, 1989.

[3] Alon, N., Bárány, I., Füredi, Z., and Kleitman, D.J.: Point selections and weak $\epsilon$-nets for convex hulls. Combin. Probab. Comput., 1:189-200, 1992.

[4] Borsuk, K.: Drei Sätze ber die n-dimensionale euklidische Sphäre, Fund. Math., 20 (1933), 177-190.

[5] Dey, T. K. and Pach, J.: Extremal Problems for Geometric Hypergraphs. In Proceedings of the 7th international Symposium on Algorithms and Computation (December 16-18, 1996).

[6] Helly, E.: Über Mengen konvexer Körper mit gemeinschaftlichen Punkten, Jber. Deutsch. Math. Vereinig. 32, 175-176 (1923).

[7] Katchalski, M. and Last, L.: On geometric graphs with no two edges in convex position, Discrete Comput. Geom. 19 (1998), no. 3, Special Issue, 399-404.

[8] Katchalski, M., Liu, A.: A problem of geometry in $\mathbb{R}^{n}$. Proc. Am. Math. Soc. 75, 284-288 (1979).

[9] Klazar, M., Marcus, A.: Extensions of the linear bound in the Füredi-Hajnal conjecture, Adv. in Appl. Math. 38 (2006), no. 2, 258-266.

[10] Kővári, T., Sós, V., Turán, P.: On a problem of K. Zarankiewicz. Coll. Math., 3:50-57, 1954.

[11] Marcus, A. and Tardos, G.: Excluded permutation matrices and the Stanley-Wilf conjecture. J. Comb. Theory Ser. A 107, 1 (Jul. 2004), 153-160, 2004.

[12] Matoušek, J.: 2002 Lectures on Discrete Geometry. Springer-Verlag New York, Inc.

[13] Marcus, A. and Tardos, G.: Excluded permutation matrices and the Stanley-Wilf conjecture, J. Combin. Theory Ser. A 107 (2004), no. 1, 153-160.

[14] Nivasch, G. and Sharir, M.: Note: Eppstein's bound on intersecting edges revisited. J. Comb. Theory Ser. A 116, 2 (Feb. 2009), 494-497.

[15] Pach, J. and Töröcsik, J.: Some geometric applications of Dilworth's theorem. In Proceedings of the Ninth Annual Symposium on Computational Geometry (San Diego, California, United States, May 18 - 21, 1993). SCG '93. ACM, New York, NY, 264-269.

[16] Pinchasi, R.: Geometric graphs with no two parallel edges. Combinatorica 28, 1 (Jan. 2008), 127-130, 2008. 
[17] Tóth, G.: Note on geometric graphs. J. Comb. Theory Ser. A 89, 1 (Jan. 2000), 126-132, 2000.

[18] Valtr, P.: Graph drawings with no $k$ pairwise crossing edges, In Graph Drawing (Rome), Lecture Notes in Computer Science, vol. 1353, 1997, pp. 205-218.

[19] Wagner. U.: $k$-Sets and $k$-Facets, Discrete and Computational Geometry - 20 Years Later (Eli Goodman, János Pach, and Ricky Pollack, eds.), Contemporary Mathematics 453, American Mathematical Society, 2008.

[20] Živaljević, R. T. and Vrećica, S. T.: The colored Tverberg's problem and complexes of injective functions. J. Comb. Theory Ser. A 61, 2 (Nov. 1992), 309-318, 1992. 